\title{
Online mutual support in bereavement: An empirical examination
}

\author{
Karolijne van der Houwen $^{\mathrm{a}, *}$, Margaret Stroebe ${ }^{\mathrm{a}}$, Henk Schut ${ }^{\mathrm{a}}$, Wolfgang Stroebe ${ }^{\mathrm{b}}$, Jan van den Bout ${ }^{\mathrm{a}}$ \\ ${ }^{a}$ Clinical and Health Psychology, Utrecht University, P.O. Box 80140, 3508 TC Utrecht, The Netherlands \\ ${ }^{\mathrm{b}}$ Social and Organizational Psychology, Utrecht University, P.O. Box 80140, 3508 TC Utrecht, The Netherlands
}

\section{A R T I C L E I N F O}

\section{Article history:}

Available online 19 June 2010

\section{Keywords:}

Bereavement

Grief

Mutual support

Internet

\begin{abstract}
A B S T R A C T
Thus far, online mutual bereavement support has not been the subject of rigorous empirical examination, despite the fact that this type of support is rapidly growing. Assessment is needed, not only to establish whether there are desirable effects, but also whether there could be negative side effects. This study was designed to increase our understanding of the people who use online mutual bereavement support and provide a preliminary examination of its potential to ameliorate their suffering. Our findings show that people who are currently using online mutual bereavement support are younger, less likely to be part of a religious community, and more likely to have lost a child than bereaved people who had never used this type of support. The former group also shows worse mental health and reports lower levels of social support than people who had stopped using online mutual bereavement support. There was no indication that people who seek out online mutual bereavement support were more likely to live in remote, rural areas than people who do not use this type of support. Using this type of support did not predict changes in mental health over time. Implications of these findings are discussed.
\end{abstract}

() 2010 Elsevier Ltd. All rights reserved.

\section{Introduction}

Mutual support approaches for bereaved people were pioneered by Silverman $(1972,1986)$ some decades ago in her well-known widow-to-widow program. The idea behind this program was that widows would identify with and receive assistance from a peer, namely, another widow who had herself been through the ordeal of bereavement. There have been numerous developments in bereavement support programs since that time, but perhaps the most significant of these has been the emergence of online mutual support groups, which has opened up many new opportunities in the provision of support. In this paper, we report an empirical study that sheds light on the characteristics of persons who use online mutual bereavement support, and that examines the impact of this type of support.

Online mutual bereavement support can best be understood in the context of online support in general. Thus, in the following part, we describe features and research findings pertaining to this broader domain. Then we review the available studies that have focused specifically on bereavement. Against this background we present our own empirical work.

\subsection{The provision of online mutual support}

Online support groups have expanded rapidly in recent years: as of April 2009 Yahoo!Groups (http://www.yahoo.com) listed over

\footnotetext{
* Corresponding author. Current address: Statistics Netherlands, P.O. Box 4481, $6401 \mathrm{CZ}$, Heerlen, The Netherlands. Tel./fax: +31 455707312.

E-mail address: hk.vanderhouwen@cbs.nl (K. van der Houwen).
}

50,000 electronic support groups in the health and wellness section, more than twice as many as 5 years ago (Eysenbach, Powell, Englesakis, Rizo, \& Stern, 2004). ${ }^{1}$ Two types of online groups can be distinguished. In synchronous groups, such as chat rooms, individuals can exchange messages in real time. In asynchronous groups, members can read and exchange messages, but are not necessarily simultaneously connected to a network. Examples of the latter include email lists and Internet forums. Registration is typically needed to gain access to chat rooms and email lists, and to post messages on Internet forums (but not to read messages on Internet forums). However, the type and extent of information required to register varies widely between groups, ranging from the provision of a valid e-mail address to extensive demographic and other details.

Online support has a number of potential advantages over traditional face-to-face support (see also Stroebe, van der Houwen, \& Schut, 2008). The Internet is not limited by geographical boundaries: it offers access to support to people living in remote areas, where help may not be readily available and to those who cannot easily leave their homes as well as to those who do not have the means (money, transportation) to engage in traditional mutual support. Similarly, it enables people suffering from rare conditions to come into contact with each other, making highly specialized groups possible. Some researchers have suggested that online groups may facilitate disclosure because its members can remain anonymous (Mo, Malik, \& Coulson, 2009). Finally, asynchronous groups offer the possibility to have contact $24 \mathrm{~h}$ a day, 7 days a week.

\footnotetext{
${ }^{1}$ It is important to keep in mind, though, that this number also includes groups that are very small (i.e. with only a few members) or inactive.
} 
The popularity of online mutual support and its potential to function as an easy accessible, low-cost resource for people experiencing difficulties have captured the interest of researchers. Consequently, many studies have been devoted to virtual health communities. Some have focused on the exchanges that take place within these groups. For example, Mo and Coulson (2008) examined the nature of social support exchanged within an online HIV/AIDS support group by analyzing 1138 messages. Other studies have surveyed members of online support groups in order to gain a better understanding of the characteristics of people using this type of support and their experiences with it. This type of research has provided information about, among others, users of a dental anxiety group (Coulson \& Buchanan, 2007), and members of psoriasis (Idriss, Kvedar, \& Watson, 2009) and infertility support groups (Malik \& Coulson, 2008).

Despite these research efforts, thus far, surprisingly little is known about the efficacy of online mutual support (Eysenbach et al., 2004). An important reason for this is that most studies that have investigated the effects of this type of support suffer from serious methodological shortcomings (for two exceptions see the research by Houston, Cooper, and Ford (2002), who examined the correlates of usage level with outcome measures, and the work of Freeman, Barker, and Pistrang, (2008), who carried out a randomized controlled trial). Some have relied solely on subjective accounts (i.e. they have asked members of support groups about their satisfaction with this type of support) making it impossible to draw firm conclusions about efficacy (demand characteristics and dropout-biases are well-established limitations of subjective accounts). Others have employed cross-sectional designs (limiting temporal inferences concerning the precise relations between factors investigated) or non-controlled before-after designs (which makes it impossible to attribute improvements of psychological or health outcomes to the intervention as many participants improve naturally over time).

The dearth of sound research on the effects of mutual online support begs the question whether it is really necessary to study this subject. Do these resources even need to actually be helpful (as long as people want to access them and they are not associated with huge burdens to individual or society)? We would argue that there are at least two reasons why it is important to investigate the efficacy of online mutual help groups: (1) to make sure that people are not actually harmed by this type of support, and (2) to assist people in making a decision whether or not to invest their time and energy in this type of support.

Of special interest with regard to the first point are some of the unique features of Internet support mentioned above: anonymity, lack of face-to-face contact, and 24/7 availability. Whereas anonymity may increase disclosure, it can also invite or facilitate inappropriate responses, perhaps even leading to exploitation or abuse by persons who enter online groups with ulterior motives. Also, the lack of physical cues makes it much more difficult to identify persons who are experiencing serious difficulties and might be in need of professional help. The asynchronous nature of most groups further complicates the possibility to identify such persons in a timely fashion. Finally, 24/7 availability might increase rather than decrease ruminative thinking and distress, and may induce wallowing in one's own problems (Shaw, Han, Hawkins, McTavish, \& Gustafson, 2008; Stroebe et al., 2008). Finally, it could encourage people to withdraw from their normal social world.

\subsection{Online mutual bereavement support}

Bereaved people do not appear to be excluded from the online support trend. On the contrary, mourning and loss support groups make up 10 percent of all electronic support groups in the health and wellness section of Yahoo! Groups alone, making bereavement the third most popular subject after diseases and conditions (43\%) and weight issues (13\%).

Bereavement researchers' involvement with online support groups so far has mainly been through their employment of these groups to recruit participants for their studies. Only a few studies have made these groups the focus of investigation. Among these, Hollander (2001) examined a large number of e-mails from a number of online support groups for survivors of the suicides of loved ones, while Capitulo (2004) investigated the content of exchanges in one particular email list for mothers who had suffered a perinatal death. Both were qualitative studies that aimed to arrive at a deeper understanding of what exactly happens within these groups, and to identify common themes. They did not provide information about the characteristics of people using this type of support nor about its effectiveness. Insight into who is using online mutual support is important as well.

In a recent cross-sectional survey study Feigelman, Gorman, Chastain Beal, and Jordan (2008) compared members of one of the largest Internet survivor of suicide support groups ("Parents of Suicide") with face-to-face support group affiliates - who had suffered a similar loss - on a large number of variables using univariate analyses. These analyses showed that Internet affiliates were more recent survivors, were more often female, younger, less educated, divorced or separated, and living alone. They also had lower incomes, were more likely to have no religious affiliation, were less likely to participate in any organized religious observances, and experienced less social support and more mental health problems (i.e. grief, depression, suicidal ideation). No differences were found on number of children or rural-urban residence. However, their results regarding the differences between these two groups should be interpreted with care: spurious results may have been reported both due to the separate analyses of a large number of variables and to the confounding effects of other variables. Feigelman et al. (2008) also examined features which support group members value about online participation and concluded that "Our data [...] bear on the question of the helpfulness of Internet support groups in enhancing the adaptation of survivors of child suicide loss" and "Internet users [...] sought and obtained valuable help from the Internet support resource" (pp. 239 and 217 ; italics added). However, there were no sound empirical bases for these claims. The study was cross-sectional in nature and lacked objective measures of gains.

To summarize, we are aware of only one study that has looked at the characteristics of online mutual bereavement support users. However, this investigation suffered from methodological shortcomings and was limited to one specialized support group. No studies to date have examined the efficacy of online mutual bereavement support.

As already explained, it is important that we increase our knowledge of online mutual bereavement support. Therefore, the current study was designed to (1) gain better understanding of the characteristics of online mutual bereavement support users and (2) examine whether using online mutual bereavement support is associated with changes in mental health over time. People currently using online mutual support were compared on a number of potentially discriminating factors to bereaved people who had never used this type of support and to bereaved people who had used this type of support in the past. The selection of these factors was based partly on the results reported by Feigelman et al. (2008) and partly on the literature on bereavement in general. We included demographics (age, gender, education level, rural-urban residence), bereavement-related variables (type of loss, cause of death, time since death, (un)expectedness of the loss), mental health variables (grief, depressive symptoms, and emotional loneliness), and support variables (professional help, social support, involvement in a religious community). All factors - except for res- 
idence - were examined simultaneously in a multivariate analysis in order to avoid reporting spurious results. This was done in an exploratory manner, given the lack of previous research. Referring to the second interest of the study, a controlled longitudinal design was employed to examine changes over time in relationship to mutual online bereavement support. In addition to the use of multivariate analyses and a longitudinal controlled design mentioned above, further improvements over previous designs are the inclusion of different types of losses and different forms of online support. These increase the generalizability of the results.

\section{Method}

\subsection{Participants}

This investigation was part of an IRB-approved randomized controlled trial with three measurement points, that looked at the efficacy of an e-mail based writing intervention for bereaved people (see van der Houwen, Schut, van den Bout, Stroebe, \& Stroebe, 2010). Only data from participants who were assigned to the no-treatment control condition were included in the present study. Because the main variable of interest (i.e. involvement in online mutual bereavement support) was not measured until the second measurement point, a further criterion for inclusion in the current investigation was that complete data were available at the second measurement point. All participants were recruited via the Internet. They were approached through websites, forums, and e-mail groups that focus on bereaved persons, and via organizations and support groups for the bereaved. Due to the worldwide accessibility of the Internet, participants did not come from a specific area or country. To be included in the study, people had to meet the following criteria at the time of registration: (1) at least 18 years of age, (2) native English speaker, (3) having experienced the death of a first-degree relative, (4) being significantly distressed by this loss. People who reported that they were suffering from severe depression, schizophrenia, psychotic episodes and/or were seriously considering ending their life were excluded from the study, as were people who suffered their loss at a very early age (and consequently had never consciously known or interacted with the person who died) and people who suffered multiple simultaneous losses. Participants were randomly assigned to receive or not receive the intervention (i.e. to the intervention or control condition, respectively). Participants assigned to the control condition were offered the opportunity to participate in the intervention after answering the last set of questionnaires. The sample consisted of 253 bereaved individuals. Background and loss characteristics are summarized in Table 1.

\subsection{Procedure}

Participants were sent e-mails inviting them to fill in questionnaires online at three points in time: immediately, 3 and 6 months after registering for the study. Questionnaires measured background and loss-related variables, aspects of mental and physical health, and personality and coping behavior. Up to two reminder e-mails were sent if participants failed to respond. Participants who did not respond to the reminder e-mails or who only filled in part of the questionnaires at a certain measurement point were not sent an invitation to fill in questionnaires at the next measurement point. The attrition rate in the group under investigation was $13 \%$. A logistic regression analysis was performed with dropout as the dependent variable in order to check for differences between completers and non-completers. Independent variables included the various predictor and outcome variables (of the regular analyses). According to the Wald criterion, both age and education
Table 1

Background and loss characteristics of the sample $(N=253)$.

\begin{tabular}{ll} 
Background characteristics & \\
Sex (N (\%)) & $16(6.3 \%)$ \\
Men & $237(93.7 \%)$ \\
Women & $42.97(10.98) ; 19-79$ \\
Age (in years) (M (SD); minimum-maximum) & \\
Education (highest level of schooling) (N (\%)) & $0(0 \%)$ \\
Primary school/elementary school & $3(1.2 \%)$ \\
Secondary school/high school (not finished) & $32(12.6 \%)$ \\
Secondary school/high school (finished) & $61(24.1 \%)$ \\
Some post-secondary school & $63(24.9 \%)$ \\
College diploma or equivalent & $53(20.9 \%)$ \\
University degree & $41(16.2 \%)$ \\
Postgraduate degree & \\
Loss characteristics & \\
Deceased is (N (\%)) & \\
Partner & $88(34.8 \%)$ \\
Child & $104(41.1 \%)$ \\
Parent & $41(16.2 \%)$ \\
Sibling & $20(7.9 \%)$ \\
Causes of death & \\
Natural causes & $175(69.2 \%)$ \\
Accident/homicide & $51(20.2 \%)$ \\
Suicide & $27(10.7 \%)$ \\
Time since loss (in years) (M (SD)) & $3.48(4.71)$ \\
$<6$ months & $30(11.9 \%)$ \\
>=6 months and <12 months & \\
>=12 months and <24 months $=2$ years and <5 years & $48(19.0 \%)$ \\
>=5 years or more & $64(25.3 \%)$ \\
& $56(22.1 \%)$ \\
\hline & $55(21.7 \%)$ \\
\hline
\end{tabular}

level reliably predicted dropout: completers were older $\left(\chi^{2}(1\right.$, $N=253)=5.52, p<.05)$ and higher educated $\left(\chi^{2}(1, N=253)=\right.$ $7.22, p<.01)$ than non-completers.

\subsection{Measurement instruments}

As will be described next, variables were selected from different time points for the purposes of this particular study.

\subsubsection{Background and loss-related variables}

While registering for the study, participants provided information about their place of residence (country, state/county, and town). At the first measurement point questions were asked about age, gender, education level (measured on a 7-point scale), time since death, formal relationship to the deceased (partner/parent/ child/sibling), cause of death (natural causes/accident or homicide/suicide), and level of unexpectedness of the death (measured on a 5-point scale, from totally expected to totally unexpected).

\subsubsection{Support variables}

Social support was assessed with a four-item scale of perceived social support. This scale asked two questions about social support from family members and the same two questions about social support from friends and relatives (a) "On the whole, how much do your [family members]/[friends and relatives] make you feel loved and cared for?" and (b) "How much are your [family members]/[friends and relatives] willing to listen when you need to talk about your worries or problems?" (Stroebe, Zech, Stroebe, \& Abakoumkin, 2005). Response categories were "a great deal”, "quite a bit", "some", "a little", "not at all", and "not applicable". Participants filled out this scale at all three points in time; however, in this article only data from the second measurement point were used. Cronbach's alpha was .91.

Professional help was measured at every time point by asking participants to indicate whether they were currently receiving assistance related to their grief from a professional. Only data from the second measurement point were used in this study. 
Involvement in a religious community was assessed at the first measurement point by asking participants whether they were a practicing member of an organized religion.

\subsection{Online mutual bereavement support}

Information was gathered at the second and third measurement point about participants' involvement in three types of online mutual bereavement support: email lists, Internet forums, and chat rooms. For each type of support participants were asked to indicate whether they had ever used this type of support, whether they were currently using this type of support, and, if so, for how many hours on average per week. Only data from the second measurement point were used in this study.

\subsubsection{Mental health variables}

All mental health variables were measured at each time point. Both data from the second and the third measurement moment were used in this study. Grief reactions were measured using nine items that were formulated on the basis of the criteria for complicated grief proposed for DSM-V (Prigerson, Vanderwerker, \& Maciejewski, 2008). Examples of items are "I have felt that moving on with my life (for example, making new friends, pursuing new interests) is difficult for me" and "I have felt emotionally numb (e.g. detached from others)". ${ }^{2}$ It has been shown that these 9 items constitute a concise way of measuring complicated grief (H. Prigerson, personal communication, March 10, 2006). Items were rated with respect to the past week on a 5-point scale ranging from never $(=1)$ to all of the time $(=5)$. Cronbach's alpha ranged from .91 to .92, and test-retest reliability was .81 .

Depressive symptoms were assessed using the Center for Epidemiological Studies-Depression Scale (CES-D; Radloff, 1977). In this study, Cronbach's alpha ranged from .93 to .95 , and test-retest reliability was .74 .

Positive mood was measured using the corresponding 10 items of the Positive Affect Negative Affect Schedule (PANAS; Watson, Clark, \& Tellegen, 1988). In this study, Cronbach's alpha ranged from .95 to .96 , and test-retest reliability was .74.

Emotional loneliness was measured using the following two items: (1) "I feel lonely even if I am with other people", and (2) "I often feel lonely" (W. Stroebe, Stroebe, Abakoumkin, \& Schut, 1997). Participants indicated their (dis)agreement with these statements on a 7 -point scale ranging from totally disagree $(=1)$ to totally agree $(=7)$. Cronbach's alpha ranged from .88 to .89 , and test-retest reliability was .67 .

\section{Results}

\subsection{Use of online mutual support}

A majority of participants indicated that they were currently involved in online mutual support or had been so in the past (see Table 2). Taking a closer look at the group of participants who were currently using online mutual support $(N=156)$, Table 2 shows that email lists and Internet forums were being used by 102 and 108 participants, respectively (65\% resp. 69\%), making them equally popular forms of support in this group. Chat rooms were only used by 19 participants (12\%). Over two-fifths of participants who were currently involved in online mutual support used more than one form of support.

Participants spent on average $7.4 \mathrm{~h}$ per week (SD = 9.0) using online mutual bereavement support. People who used two or three forms of support spent more hours using online support $(M=11.1$, $\mathrm{SD}=11.0)$ than people who used only one form of support $(M=4.6$,

\footnotetext{
${ }^{2}$ All materials can be obtained from the first author.
}

Table 2

Use of online mutual bereavement support at the second time point $(N=253)$.

\begin{tabular}{ll}
\hline Are currently using online mutual bereavement support & $156(61.7 \%)$ \\
Email list & $42(16.6 \%)$ \\
Internet forum & $45(17.8 \%)$ \\
Chat room & $3(1.2 \%)$ \\
Email list + Internet forum & $50(19.8 \%)$ \\
Email list + chat room & $3(1.2 \%)$ \\
Internet forum + chat room & $6(2.4 \%)$ \\
Email list + Internet forum + chat room & $7(2.8 \%)$ \\
Have used online mutual bereavement support in the past & $47(18.6 \%)$ \\
Have never used online mutual bereavement support & $50(19.8 \%)$ \\
\hline
\end{tabular}

$\mathrm{SD}=6.8)(t(154)=-4.42, p<.001)$. The type of support did not appear to be associated with the number of hours spent online using it: participants who exclusively used email lists $(N=42)$ spent as much time online as people who exclusively used Internet forums $(N=45)$, $t(85)=-0.20, p=0.84(M=4.0, \mathrm{SD}=5.1$ resp. $M=4.2, \mathrm{SD}=4.8)$.

\subsection{Predictors of current and past use of online mutual support}

Two logistic regression analyses were performed, one in which we checked for differences between people who had never used online mutual bereavement support and those who were currently using it, and one in which we checked for differences between people who had been using this type of support in the past and those who were currently using it. Predictors were demographics (age, gender and education level), bereavement-related factors (time since death, formal relationship to the deceased, cause of death, and expectedness of the death), support variables (social support, professional help, involvement in a religious community) and mental health (grief, depressive symptoms, and emotional loneliness). The results of the analyses can be found in Table 3.

Participants who were currently using online mutual bereavement support differed from participants who had never used this type of support in a number of respects: the first group was younger, more likely to have lost a child (versus a parent or sibling) and less likely to be part of a religious community. Differences were also found between participants who had stopped using online mutual bereavement support and participants who were still using it: the latter group showed worse mental health (i.e. higher levels of grief and emotional loneliness) and reported lower levels of social support.

Additionally, in order to gain more insight into the stable characteristics of online mutual bereavement support users, a new group was created in which we combined people who were currently using online bereavement support with people who had been using this type of support in the past. Using logistic regression analysis, this group was compared to the group of people who had never used this type of support on relevant background (age, gender, education level, involvement in a religious community) and loss-related variables (type of loss, cause of death, (un)expectedness of death, time since death). The results of this analysis were very similar to the one presented earlier in which we compared participants who were currently using online mutual bereavement support with participants who had never used this type of support: online mutual bereavement support users were younger $(p<.10)^{3}$, were more likely to have lost a child (than a parent or a sibling; $p<.001$ and $p<.10$, respectively), and were less likely to be part of a religious community $(p<.01) .{ }^{3}$ The analysis also showed that support users were more likely to be female $(p<.01)$.

Another variable that has been associated with the use of online mutual support is residence in rural (compared to urban) areas. Be-

\footnotetext{
${ }^{3}$ Additional analyses clarified that the effect of age was reduced to a trend due to
} overlapping variances between gender and age. 
Table 3

Predictors of current and past use of online mutual bereavement support.

\begin{tabular}{|c|c|c|c|c|}
\hline & \multicolumn{2}{|c|}{ Never used $(=0)$ versus current use $(=1)$} & \multicolumn{2}{|c|}{ Used in the past $(=0)$ versus current use $(=1)$} \\
\hline & B & SE & B & SE \\
\hline \multicolumn{5}{|l|}{ Demographics } \\
\hline Age & $-0.041^{*}$ & 0.020 & -0.007 & 0.020 \\
\hline Gender ( 0 = male, 1 = female $)$ & 1.185 & 0.738 & -20.839 & 12459.925 \\
\hline Education level & 0.030 & 0.150 & -0.189 & 0.163 \\
\hline \multicolumn{5}{|l|}{ Bereavement-related factors } \\
\hline Time since death & 0.041 & 0.044 & 0.110 & 0.063 \\
\hline \multicolumn{5}{|l|}{ Kinship $(0=$ child $)$} \\
\hline Partner & -0.347 & 0.568 & -0.413 & 0.512 \\
\hline Parent & $-2.843^{* * *}$ & 0.066 & -1.186 & 0.633 \\
\hline Sibling & $-1.626^{*}$ & 0.741 & -0.987 & 0.802 \\
\hline \multicolumn{5}{|l|}{ Cause of death ( $0=$ natural causes $)$} \\
\hline Accident/homicide & -0.479 & 0.574 & 0.678 & 0.647 \\
\hline Suicide & -0.165 & 0.668 & 0.427 & 0.649 \\
\hline Expectedness of death & -0.018 & 0.158 & -0.113 & 0.151 \\
\hline \multicolumn{5}{|l|}{ Support variables } \\
\hline Social support & -0.336 & 0.223 & $-0.671^{* *}$ & 0.217 \\
\hline Professional help $(0=$ no help $)$ & 0.470 & 0.459 & 0.370 & 0.429 \\
\hline Involvement in a religious community $(0=$ no $)$ & $-1.162^{* *}$ & 0.433 & 0.460 & 0.426 \\
\hline \multicolumn{5}{|l|}{ Mental health } \\
\hline Grief & 0.043 & 0.042 & $0.081^{*}$ & 0.041 \\
\hline Depressive symptoms & -0.029 & 0.030 & -0.024 & 0.030 \\
\hline Emotional loneliness & 0.101 & 0.079 & $0.179^{*}$ & 0.077 \\
\hline
\end{tabular}

Table 4

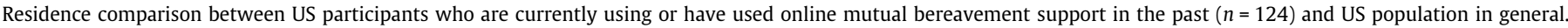

\begin{tabular}{|c|c|c|c|}
\hline Code & Description & Sample & United States \\
\hline 1 & County in metro area with 1 million population or more & $62(50.0 \%)$ & $149224067(53.0 \%)$ \\
\hline 2 & County in metro area of 250,000 to 1 million population & $18(14.5 \%)$ & $55514159(19.7 \%)$ \\
\hline 3 & County in metro area of fewer than 250,000 population & $19(15.3 \%)$ & $27841714(9.9 \%)$ \\
\hline 4 & Nonmetro county with urban population of 20,000 or more, adjacent to a metro area & $8(6.5 \%)$ & $14442161(5.1 \%)$ \\
\hline 5 & Nonmetro county with urban population of 20,000 or more, not adjacent to a metro area & $1(0.8 \%)$ & $5573273(2.0 \%)$ \\
\hline 6 & Nonmetro county with urban population of $2500-19999$, adjacent to a metro area & $8(6.5 \%)$ & $15134357(5.4 \%)$ \\
\hline 7 & Nonmetro county with urban population of $2500-19999$, not adjacent to a metro area & $7(5.6 \%)$ & $8463700(3.0 \%)$ \\
\hline 8 & Nonmetro county completely rural or less than 2500 urban population, adj. to metro area & $1(0.8 \%)$ & $2425743(0.9 \%)$ \\
\hline 9 & Nonmetro county completely rural or less than 2500 urban population, not adj. to metro area & $0(0 \%)$ & $2802732(1.0 \%)$ \\
\hline
\end{tabular}

cause participants in our study came from different countries we were unable to use one system for categorizing participants' place of residence, and therefore could not include this variable in the logistic regression analyses. We were, however, able to check this hypothesis for a subset of our sample, namely, participants living in the United States $(N=156)$, by using the 2003 Rural/Urban Continuum Codes from the Economic Research Service (ERS) of the Department of Agriculture. Of all participants living in the United States, 152 (97\%) could be classified according to this system. Of this group, 124 persons had used online mutual bereavement support in the past or were still using it. Their residence data were compared to those of the entire population of the United States (see Table 4). Contrary to what is sometimes claimed, people who seek out online mutual bereavement support are not more likely to live in remote, rural areas than the average Northern American. .

\subsection{Changes in mental health over time in relationship to online mutual bereavement support}

Next, we checked whether making active use of online mutual bereavement support was associated with changes in people's

\footnotetext{
${ }^{4}$ of course, we are aware of the fact that our US subgroup is not representative of the North American population in general. However, we have no reason to assume that our group (mostly younger women) would have very different residence patterns from the rest of the population.
}

mental health over time (i.e. between the second measurement moment when online activity was measured and the third measurement moment 3 months later). For each of the mental health variables (grief, depressive symptoms, emotional loneliness and positive mood) a model was constructed containing the mental health variable measured at the third measurement moment as a dependent variable and the following predictors: the same mental health variable measured at the second measurement moment, use of online mutual bereavement support at the second measurement moment (yes or never), age, formal relationship to the deceased (dummy coded), involvement in a religious community, and education level. ${ }^{5}$ Age, formal relationship to the deceased and involvement in a religious community were added to the model to control for differences between participants who had never used online mutual bereavement support at the second measurement moment and those who were using this type of support at that time. Education level was added, because it predicted dropout between the second and third measurement point (as did age, but this variable was already part of the model). The model was tested using AMOS 7.0, because this program does not assume equal numbers of observations, which

\footnotetext{
${ }^{5}$ We considered adding the number of hours spent on average per week using online mutual bereavement support at the second measurement point as a predictor in the model. However, we decided against this because in a separate analysis no relationship was found between the number of hours spent online and change in mental health.
} 
Table 5

Mean scores of the outcome measures (SD in parentheses) for online support users and non-users.

\begin{tabular}{lcclll}
\hline & \multicolumn{2}{l}{ Online support users } & & Non-users & \\
\cline { 2 - 3 } \cline { 5 - 6 } \cline { 5 - 6 } & $t=2$ & $t=3$ & & $t=2$ & $t=3$ \\
\hline Grief & $24.9(8.8)$ & $22.7(8.8)$ & & $21.5(8.4)$ & $20.1(8.8)$ \\
Depressive symptoms & $22.8(13.4)$ & $20.6(13.6)$ & & $20.9(12.0)$ & $18.5(13.3)$ \\
Emotional loneliness & $9.4(3.9)$ & $8.2(3.9)$ & & $8.0(3.9)$ & $8.2(4.3)$ \\
Positive mood & $27.2(9.8)$ & $28.6(9.5)$ & & $29.2(9.9)$ & $30.2(10.6)$ \\
\hline
\end{tabular}

means that all cases can remain in the analyses, thereby increasing the precision of the estimates and the power of the statistical tests. Table 5 gives means and standard deviations for the outcome measures.

Using online mutual bereavement support did not predict changes in mental health over time. No differences were found on grief $(b=0.819, t=0.855, p=.39)$, depressive symptoms $(b=$ 1.833, $t=1.070, p=.29)$, emotional loneliness $(b=-0.724, t=$ $-1.265, p=.21)$ or positive $\operatorname{mood}(b=0.819, t=0.855, p=.39)$.

\section{Discussion}

Thus far, online mutual bereavement support has not been the subject of rigorous empirical examination. This is unfortunate, because some of the characteristics, which make online support so attractive, may have negative side effects (so we should make sure that people are not actually harmed by this type of support), and because it appears to be an increasingly popular support method (making it important to understand who participates in this type of support). This study was designed first, to increase our understanding of the people who use online mutual bereavement support and second, to provide a preliminary examination of its potential to ameliorate their suffering. With respect to the first interest, our findings showed a number of differences between people who were currently using online support and people who had never used this type of support as well as between the former group and people who had used this type of support in the past. With respect to the second concern, the use of online mutual bereavement support was not associated with changes in mental health over time. We next review our findings in more detail and address some of the limitations of our study. Finally, we offer some suggestions for further research.

We found that a majority of participants were currently involved in online mutual support or had been so in the past. This is not surprising, given the fact that our sample was partly acquired through email lists and Internet forums for the bereaved. Thus, the likelihood of using online mutual bereavement support in this study is likely to be different from that found among the bereaved in general. Whereas email lists and Internet forums were both popular forms of support, only a small minority reported using chat rooms. Although this may partly be due to the fact that we did not use chat rooms to recruit our participants, it could indeed represent a true preference for asynchronous groups: research shows that 24/7 availability is one of the features of online support that is most appreciated by people (e.g. Feigelman et al., 2008).

Our results showed that a great deal of time was spent interacting on the Internet: more than $1 \mathrm{~h}$ per day on average. This suggests that online support takes a prominent place in the lives of these bereaved people. This concurs with findings reported by Feigelman et al. (2008), who found that approximately half of all respondents spent six or more hours weekly participating in the Internet support group ("Parents of Suicide") they investigated. Our replication of their data is especially noteworthy, because we included different types of losses and different forms of online support. Furthermore, we extended their findings by showing that a large minority of respondents used more than one form of support and that using more forms of support increased the time spent online.

Our study revealed a number of features of online support users: Participants who were currently using online mutual bereavement support or had done so in the past were more likely to be female, younger, less likely to be part of a religious community, and more likely to have lost a child (than a parent or a sibling) than people who had never used this type of support. Again, this concurs with results reported by Feigelman et al. (2008) who also found that "more women, younger survivors, and those less connected to conventional religious observances were over-represented" (p. 239) in Internet affiliates. The effect of age may well reflect a pattern found among the non-bereaved, showing an overall decline in usage of interactive web services as age increases (http://spire.conted.ox.ac.uk/trac_images/spire/SPIRESurvey.pdf). With regard to involvement in a religious community: it is perhaps not surprising that people who are not part of a religious community would seek out another, online, community for sharing thoughts and feelings. However, as with age, this phenomenon need not be bereavement specific.

Our finding that people who have lost a child have a higher chance of being involved in online mutual support could be attributed to the fact that there appear to be more groups available online of parents that suffered the loss of a child: groups that focus on child loss constitute $23 \%$ of all mourning and loss support groups in the health and wellness section of Yahoo!Groups (only 12\% focus on parent loss). Of course, this does not answer the question why this type of loss is overrepresented online (i.e. people are far more likely to lose their parents or a sibling than a child). One reason for this may be that parents who have lost a child are less likely than people who have lost a parent or a sibling to encounter fellow sufferers in their social network. The Internet facilitates meeting and interacting with such people. It could also reflect the nature of child loss, often claimed to be the worst type of loss that can occur. Parents may feel the need for support over a longer period of time, when others around them may no longer be so forthcoming with their support.

It is interesting to note that the two groups described before (those currently using online mutual support versus those who had never used this type of support) did not differ with respect to professional or social support. This suggests that online mutual bereavement support is not used as a substitute for these forms of support, but rather as an addition to them. This notion is further supported by the fact that people who seek out online mutual bereavement support are not more likely to live in remote, rural areas (where professional help may not be readily available). However, given the cross-sectional nature of this analysis, other explanations may be possible as well. For example, it may be that people start using online support because they are dissatisfied with the help they are getting from family and friends but that their perception of this help subsequently changes. Longitudinal research is needed to further explore these issues.

Differences were also found between people who had stopped using online mutual bereavement support and those who were still using it: the former group showed better mental health (i.e. lower levels of grief and emotional loneliness) and reported higher levels of social support. Although it makes sense that people would stop using online support once they start feeling better, again the cross-sectional nature of the analysis precludes this temporal inference: it could also be the case that people started feeling better after they stopped using online support. In order to better understand this relationship, it is important to look at 
the changes in mental health over time in relationship to online mutual bereavement support. Fortunately, our longitudinal dataset enabled us to do this.

Using online mutual bereavement support was not associated with changes in mental health over time. No differences were found on grief, depressive symptoms, emotional loneliness or positive mood. One reason for this could be that the follow-up period (i.e. 3 months) that we used was too short for changes to become evident. Another possibility might have to do with the fact that we do not know anything about the time people have been using this type of support. Changes in mental health could occur only when people first start, but not over longer durations. However, it could also be the case that the lack of findings is simply in line with research showing that social support does not appear to accelerate the process of recovery from bereavement (Stroebe et al., 1997, 2005).

In discussing our findings, we have already addressed some limitations of our study. A point we have not yet touched upon is the fact that all our participants - including the ones who never used online mutual bereavement support - were Internet savvy: they registered for this study via our website, we were in contact with them via e-mail and they completed questionnaires electronically. One could argue that this has biased our results regarding the differences between those who use and those who have never used online mutual bereavement support. We do not hold this view; rather, we feel that this was a strong point of our study, because it enabled us to attribute any differences we found to a true preference for online mutual bereavement support instead of to a lack of Internet knowledge or access. However, this does not mean that we assume representativeness. Selective participation is, unfortunately, quite common in bereavement research (Stroebe \& Stroebe, 1989). It remains critically important to acknowledge and to assess the significance of potential biases associated with selection in all investigations.

Our study provides valuable information about users of online mutual bereavement support and it is the first to shed light on mental health changes associated with this type of support. A number of methodological improvements were introduced. Future research should focus on replicating and further exploring our findings. First, longitudinal studies are needed that measure online activity at multiple points in time. This way, one can examine what predicts the commencing and termination of online support use. It also enables one to determine changes over longer periods of time. Second, it is possible that online mutual bereavement support only benefits (or harms) certain groups of people. Research should focus on identifying such moderating variables. Finally, although there appear to be a lot of online mutual support groups available to the bereaved, at this moment nothing is known about the percentage of bereaved that use online mutual bereavement support. Herein lies a major task for future researchers.

\section{References}

Capitulo, K. L. (2004). Perinatal grief. MCN The American Journal of Maternal/Child Nursing, 29, 305-311.

Coulson, N. S., \& Buchanan, H. (2007). Self-reported efficacy of an online dental anxiety support group: A pilot study. Community Dentistry and Oral Epidemiology, 36, 43-46.

Eysenbach, G., Powell, J., Englesakis, M., Rizo, C., \& Stern, A. (2004). Health related virtual communities and electronic support groups: Systematic review of the effects of online peer to peer interactions. British Medical Journal, 328, 1166-1170.

Feigelman, W., Gorman, B. S., Chastain Beal, K., \& Jordan, J. R. (2008). Internet support groups for suicide survivors: A new mode for gaining bereavement assistance. Omega, 57, 217-243.

Freeman, E., Barker, C., \& Pistrang, N. (2008). Outcome of an online mutual support group for college students with psychological problems. Cyberpsychology $\mathcal{E}$ Behavior, 11, 591-593.

Hollander, E. M. (2001). Cyber community in the valley of the shadow of death. Journal of Loss and Trauma, 6, 135-146.

Houston, T. K., Cooper, L. A., \& Ford, D. E. (2002). Internet support groups for depression: A 1-year prospective cohort study. American Journal of Psychiatry, 159, 2062-2068.

Idriss, S. Z., Kvedar, J. C., \& Watson, A. J. (2009). The role of online support communities: Benefits of expanded social networks to patients with psoriasis. Archives of Dermatology, 145, 46-51.

Malik, S. H., \& Coulson, N. S. (2008). Computer-mediated infertility support groups: An exploratory study of online experiences. Patient Education and Counseling, 73, 105-113.

Mo, P. K. H., \& Coulson, N. S. (2008). Exploring the communication of social support within virtual communities: A content analysis of messages posted to an online hiv/aids support group. Cyberpsychology and Behavior, 11, 371-374.

Mo, P. K. H., Malik, S. H., \& Coulson, N. S. (2009). Gender differences in computermediated communication: A systematic literature review of online healthrelated support groups. Patient Education and Counseling, 75, 16-24.

Prigerson, H. G., Vanderwerker, L. C., \& Maciejewski, P. K. (2008). A case for inclusion of prolonged grief disorder in DSM-V. In M. Stroebe, R. Hansson, H. Schut, \& W. Stroebe (Eds.), Handbook of bereavement research and practice. 21st century perspectives (pp. 165-186). Washington, DC: American Psychological Association.

Radloff, L. S. (1977). The CES-D Scale: A self-report depression scale for research in the general population. Applied Psychological Measurement, 1, 385-401.

Shaw, B. R., Han, J. Y., Hawkins, R. P., McTavish, F. M., \& Gustafson, D. H. (2008). Communicating about self and others within an online support group for women with breast cancer and subsequent outcomes. Journal of Health Psychology, 13, 930-939.

Silverman, P. R. (1972). Widowhood and preventive intervention. The Family Coordinator, 21, 95-102.

Silverman, P. R. (1986). Widow-to-widow. New York: Springer.

Stroebe, M., \& Stroebe, W. (1989). Who participates in bereavement research? A review and empirical study. Omega, 20, 1-29.

Stroebe, M., van der Houwen, K., \& Schut, H. (2008). Bereavement support, intervention, and research on the Internet: A critical review. In M. Stroebe, $R$. Hansson, H. Schut, \& W. Stroebe (Eds.), Handbook of bereavement research and practice. 21st century perspectives (pp. 551-574). Washington, DC: American Psychological Association.

Stroebe, W., Stroebe, M., Abakoumkin, G., \& Schut, H. (1997). The role of loneliness and social support in adjustment to loss: A test of attachment versus stress theory. Journal of Personality and Social Psychology, 70, 1241-1249.

Stroebe, W., Zech, E., Stroebe, M. S., \& Abakoumkin, G. (2005). Does social support help in bereavement? Journal of Social and Clinical Psychology, 24, 1030-1050.

van der Houwen, K., Schut, H., van den Bout, J., Stroebe, M., \& Stroebe, W. (2010). The efficacy of a brief internet-based self-help intervention for the bereaved. Behaviour Research and Therapy, 48, 359-367.

Watson, D., Clark, L., \& Tellegen, A. (1988). Development and validation of brief measures of positive and negative affect: The PANAS scales. Journal of Personality and Social Psychology, 54, 1063-1070. 